\title{
Effect of Haze on Human Health and Prevention
}

\author{
Hao Chen ${ }^{1, a}$, Shubing Liu ${ }^{2, b}$ and Yingchao Lin ${ }^{3, c^{*}}$ \\ ${ }^{1,2}$ Department of Social Psychology, Zhou Enlai School of Government, \\ Nankai University, Tianjin, China \\ ${ }^{3}$ Centre for Urban Transport Emission Research, College of Environmental Science and \\ Engineering, Nankai University, Tianjin 300071, China \\ ahul11919@gmail.com, bliushubing05@gmail.com, dei@nankai.edu.cn
}

Keywords: haze; physical health; mental health; PM2.5; air pollution

Abstract. China has witnessed several large-scale fog and haze outbursts in recent years. These phenomena can jeopardize human physical and mental health by causing respiratory diseases or depression. They also aroused extensive social discussion among all levels in the country. This paper briefly reviews the definition and causes of fog and haze, discusses the threats they pose to human health, and gives proposals on how to prevent them.

\section{Haze and Its Causes}

Differences between Fog and Haze. Haze typically occurs in central-east China in autumn and winter, results in large areas of low visibility weather phenomena. It includes two kinds of visual obstacles, fog and haze.

Fog is an aerosol system formed by a large amount of tiny water droplets or ice crystals suspending in the atmosphere at or near the earth's surface. It results from water vapor condenses into tiny liquid water droplets suspended in the air. Fog can reduce air transparency and deteriorate visibility. When the visibility was reduced to less than $1 \mathrm{~km}$, such meteorological phenomenon is defined as fog [1]. Fog can be considered as a particular form of cloud, it can be called low-lying cloud.

Haze consists of matters such as sulfuric acid, dust, nitric acid and organic hydrocarbons suspending in the air, which make turbid atmosphere and visibility deterioration. The visual obscuration caused by non-aqueous aerosol systems is defined as haze or dust-haze when visibility is less than $10 \mathrm{~km}$. Generally, the diurnal variation of haze is not obvious. When the air mass is relatively stable, haze can last for a long period, sometimes over 10 days.

Fog consists of water vapor or suspending micrometer-scale water droplets. Haze is a type of air turbidity due to large amounts of suspending smoke and dust particles. Fog is a natural weather phenomenon while haze is a pollutant. Fog normally occurs at a relative humidity near $100 \%$, which means saturated water vapor is likely to facilitate the formation of fog. However, the formation of haze only requires a certain density of particles [2]. Fog is relatively clean, but with large amounts of pollutants in the air, the water droplets are likely to absorb many hazardous substances and affect people's health ${ }^{[3]}$.

\section{The Threats Posed by Haze to Human Physical and Mental Health}

Threats to Human Physical Health. Haze contains a variety of particles harmful to the human body. Particles in the air can be divided into three categories: TSP (Total Suspended Particle), PM10 and PM2.5. Compared to PM10 and other particles, PM2.5 has a smaller diameter and a larger specific surface area (SSA). It can absorb organic and inorganic hazardous matters of various kinds, carry them in to the alveolus when entering the blood circulation system, and pose further threats to the human body.

Threats to Human Respiratory Systems. Having absorbed hazardous gases, PM2.5 may cluster certain tissues and stimulate or corrode the alveolar walls once they enter the lungs. The long-term effect can damage the defence function of the respiratory system. Long exposure to haze may cause 
bronchitis, laryngitis, pneumonia, asthma, rhinitis, conjunctivitis and allergic diseases. Riva et al[4]. conducted an experiment to male mice with $30 \mu \mathrm{L}$ of saline containing $5 \mu \mathrm{g}$ and $15 \mu \mathrm{g}$ of PM2.5 respectively for 24 hours. It was observed that a high concentration of PM2.5 could significantly increase lung impedance and alveolar collapse, as well as lung tissue inflammation, oxidative stress and damage to the mice. The reactions above are less significant on mice exposed to low-concentration PM2.5. The results reveal that the effect of PM2.5 on lung tissues had a certain dependency on dose. The research of Pope et al [5]. has proved that both long and short-time exposure to high concentrations of particulate matters can increase the morbidity and mortality of respiratory diseases in the population.

Threats to Human Cardio-cerebral Vascular Systems. The PM2.5 in haze may cause diseases as arrhythmia, coronary heart disease, myocardial infarction, acute atherosclerosis, and congestive heart failure. Velten et al. ${ }^{[6]}$ have discovered that exposure to PM2.5 during foetal and postnatal periods of mice could cause deficiencies in heart function and heart failures in adulthood. This perinatal PM2.5 injury can lead to adult heart disease. Other studies have shown that short-time exposure in high condensations of PM2.5 (even for several hours) is enough to increase heart failure hospitalisation and heart failure mortality events of the population [7-8].

Haze and Cancer. During the past 30 years, the smoking rate in our country has decreased in a constant manner whereas the lung cancer incidence rate has increased more than four times. A certain correlation might exist between the growing number of hazy. A research for Air Pollution Effects used data from 17 cohort studies based in nine European countries has revealed that, the risk of lung cancer increased by $18 \%$ for every additional $5 \mu \mathrm{g} / \mathrm{m} 3$ of PM2.5 and by $22 \%$ for every additional $10 \mu \mathrm{g} / \mathrm{m}^{3}$ of PM10. The longer the pollution lasts, the larger its likelihood to lead to lung cancer [9].

Other Threats to Human Body. Haze lowers the ultraviolet in the atmosphere near the surface of the earth, enhances the activity of infectious bacteria in the air and increases the spread of infectious diseases. Besides, after inhaled by parent, PM2.5 may result in systemic oxidative stress, inflammations and changes in hemorheology and kinetics, harms the foetus and leading to a series of negative consequences in childbirth.

\section{Haze Damage Human Mental Health.}

Haze Cause Negative Emotions. High levels of pollutants in the air may affect people's mood. Both short-term and long-term exposure to air pollution (especially pollutants produced by the fossil fuel) can increase the level of negative emotions, such as anxiety, stress and depression [10]. Wang Lixin [11] has discussed the relationship between air pollution with anxiety and depression. The results proved a positive correlation between the concentration of PM10 and the detection rate of anxiety and a negative correlation between PM2.5 and $\mathrm{O} 3$ concentration and the detection rate of depression. Haze will make people feel oppressive, anxiety, and even suffer from depression disorders.

Haze Reduce Subjective Well-Being. Welsch ${ }^{[12]}$ first measured the air quality by the amount of air pollutants such as sulfur dioxide $\left(\mathrm{SO}_{2}\right)$ and nitrogen dioxide $\left(\mathrm{NO}_{2}\right)$ in the air. The results showed that air pollution is an important reason that made differences on happiness levels among countries. After the control of the income level, research showed that air pollution can decrease the level of subjective well-being [13]. Other study has come to the same conclusion that residents in the areas with higher levels of $\mathrm{SO}_{2}$, smoke and dust emission have a proved lower level of happiness index [14]. Haze may affect people's life satisfaction and lower the subjective well-being.

\section{Measures to Prevent Haze}

Nowadays, haze has become the chief culprit that affecting the air quality of big cities. Hazardous chemical in haze may lead to asthma, bronchitis, pharyngitis and some allergic syndrome. To healthy people, haze may cause relatively minor airway injury, and symptoms such as cough, phlegm and throat pain. Thus, it is necessary for the following measures to be taken: 
First, regulate personal mood. People who are psychologically vulnerable or suffering from mental disorders are more likely to feel heavy-hearted, nervous and depressed on smoggy days. Therefore, it is important for them to regulate their moods.

Second, do not shut windows tightly. Locking the doors and windows on a hazy day is not the best choice. Without sufficient ventilation, the unclean indoor air, containing kitchen fumes and furniture additives, may also harm one's health. It is advised to open windows at noon when there's enough sunshine and fewer pollutants. for a short ventilation period,

Third, reduce the time of outdoor activities, and wear masks capable of blocking the pollutants to prevent the respiratory system from being damaged by the haze outdoors. The masks should be changed frequently. The face and other parts of the skin exposed to the smog should be washed after returning from outdoors. It is also suggested to rinse the nasal with water.

Fourth, avoid doing sports outdoors on haze days. Overexposure to the polluted air may cause not only diseases of the respiratory system, but also cardio-cerebral vascular diseases.

Fifth, according to the Air Quality Health Index (AQHI) proposed by the Healthy Environments and Consumer Safety Branch in Canada [15], China's environmental authorities can introduce a "Haze Health Index" to visually inform the public of the extent of the harm that air pollution may cause to their health, in order to guide people to take appropriate action to reduce health threats from haze.

\section{Acknowledgements}

The research is supported by Major Project of National Social Science Foundation Grant 12\&ZD218, National Social Science Foundation Grant 15BSH034, Natural Science Foundation of Tianjin 15JCQNJC15200 and Key Technologies R \& D Program of Tianjin 16YFZCSF00410.

\section{References}

[1] WU Dui. Hazy weather research in China in the last decade: A review [J]. Journal of Environmental Science, 2012, 32(2):257-269. (in Chinese)

[2] Pan H L, Wen Z S, Huang Y C, et al. Down-regulation of microRNA-144 in air pollution-related lung cancer[J]. Scientific Reports, 2015, 5. (in Chinese)

[3] Eileen Chang, Qin Tao. Some problems in the implementation of the new ground meteorological observation standard [J]. Shandong meteorology, 2006, 26 (1). (in Chinese)

[4] Zhao C X, Wang Y Q, Wang Y J, et al. [Temporal and spatial distribution of PM2.5 and PM10 pollution status and the correlation of particulate matters and meteorological factors during winter and spring in Beijing].[J]. Environmental Science, 2014, 35(2):418-27. (in Chinese)

[5] Riva D R, Magalhães C B, Lopes A A, et al. Low dose of fine particulate matter (PM2.5) can induce acute oxidative stress, inflammation and pulmonary impairment in healthy mice. [J]. Inhalation Toxicology, 2011, 23(5):257-67.

[6] Gorr M W, Velten M, Nelin T D, et al. Early life exposure to air pollution induces adult cardiac dysfunction[J]. American Journal of Physiology Heart \& Circulatory Physiology, 2014, 307(9):1353-60.

[7] Shah A S, Langrish J P, Nair H, et al. Global association of air pollution and heart failure: a systematic review and meta-analysis[J]. Lancet, 2013, 382(9897):1039-48.

[8] Link M S, Luttmann-Gibson H, Schwartz J, et al. Acute exposure to air pollution triggers atrial fibrillation. [J]. Journal of the American College of Cardiology, 2013, 62(9):816-25.

[9] Raaschou-Nielsen O, Andersen Z J, Beelen R, et al. Air pollution and lung cancer incidence in 17 European cohorts: prospective analyses from the European Study of Cohorts for Air Pollution Effects (ESCAPE)[J]. Lancet Oncology, 2013, 14(9):813-22. 
[10]Cho, J., Choi, Y. J., Suh, M., Sohn, J., Kim, H., \& Cho, S. K., et al. (2014). Air pollution as a risk factor for depressive episode in patients with cardiovascular disease, diabetes mellitus, or asthma. Journal of Affective Disorders, 157(3), 45-51.

[11] Wang L.X., Yao X.H., Jia H.Y, et al. The relationship between anxiety and depression of college students and air pollution [J]. Chinese School Health, 2016, 37(7):1036-1038. (in Chinese)

[12] Welsch H. Environment and Happiness: Valuation of Air Pollution Using Life Satisfaction Data[J]. Ecological Economics, 2006, 58(4):801-813.

[13] Welsch H. Preferences over Prosperity and Pollution: Environmental Valuation based on Happiness Surveys[J]. Kyklos, 2002, 55(4):473-94.

[14]Huang Y.M, He L.Y. (2013). City, environmental pollution and subjective well-being: evidence from Chinese. Chinese soft science (12), 82-93. (in Chinese)

[15] Stieb D M, Burnett R T, Smithdoiron M, et al. A new multipollutant, no-threshold air quality health index based on short-term associations observed in daily time-series analyses. [J]. Air Repair, 2008, 58(3):435-50. 